\title{
Perceived Impact of Social Media on Higher Secondary School Students
}

\author{
Sanyogita ${ }^{1}$, Poonam Kundu ${ }^{1}$ and Aman $^{2 *}$ \\ ${ }^{1}$ Extension Education and Communication Management, ${ }^{2}$ Farm Machinery and Power \\ Engineering, CCSHAU, Hisar, India \\ *Corresponding author
}

\section{A B S T R A C T}

\begin{tabular}{|l|}
\hline K e y w o r d s \\
Social media, \\
Communication, \\
Social apps, \\
Internet, \\
Utilization, \\
Attitude, Impact \\
\hline Article Info \\
\hline $\begin{array}{l}\text { Accepted: } \\
\text { 04 September } 2020 \\
\text { Available Online: } \\
\text { 10 October } 2020\end{array}$ \\
\hline
\end{tabular}

Social media has exploded as a category of online discourse where people create content, share it, bookmark it and network at a prodigious rate. The comparative study was conducted between four rural v/s urban schools (two private and two government higher secondary schools) of Hisar district of Haryana. The results highlighted the facts that rural parents and teachers had unfavorable attitude towards social media whereas teachers had favorable attitude towards social media. In case of urban area majority of parents and teachers both had favorable attitude towards social media. Relationship between dependent and independent variables showed age was positively correlated with physical and social health of the students whereas education was positively correlated with all the three parameters viz; mental, physical and social health of the students. This showed that young and educated person is more conscious and vigilant regarding the mental, physical and social health of their children. Most common constraints felt by the students were; parents disapproval, non-availability of net facilities and perceived internet as a costly affair. Hence in order to solve the above mentioned problems it is recommended that parents should themselves equipped with computer skill so that they can guide their children properly in terms of how, when and what type of social media they should use in order to show the right path to their children.

\section{Introduction}

Social media has exploded as a category of online discourse where people create content, share it, bookmark it and network at a prodigious rate. Because of its ease use, speed and reach, social media is fast changing the public discourse in society and setting trends and agenda in topics that range from the environment and politics to technology and the entertainment industry (Asur and Huberman, 2010). In the last ten years, the online world has changed dramatically; young men and women now exchange the ideas, feelings, personal information, pictures and videos at truly astonishing rate (Oberst, 2010). In a study conducted by Madden et al., (2013) around the world more than eighty five per cent youth spending large amount of time on internet. According to Kaur and Bashir (2015) positive as well as negative impact of social media is that to make users getting addicted and affect their academic performance. Positive effects include 
socialization, enhanced communication, learning opportunities and access to health information. Jane (2011) evaluate in their study Social media presented a virtual, unreachable world in which other children and teens might find an easy platform for cyber-bullying, particular sexual badgering. Add to that risk of a possible encounter with a sexual predator, and suddenly the world of social media seems a very dangerous place (Lou et al., 2012; Prabandari and Yuliati, 2016; Malathy and Rani, 2018).

Shahzad (2015) also found that more than ninety per cent of college students used social networks, and there was a strong link between social networking websites and student's academic achievement. Some students are not always have media literacy skills which can help them to effectively analyze, understand, And evaluate new forms of information and make smart decisions about its Quality and uses. Teenagers and young adults have especially embraced these sites as way to connect with their peers, share information reinvent their personalities, and showcase their social lives (Boyd, 2007; Camilia et al., 2013). Thustone (1946) defined attitude as the degree of positive or negative affect associated with some psychological object. By psychological object anybody meant any symbol, phrase, idea etc. towards which people can differ with respect to positive or negative affect (Kimberly, 2017; Kumar, 2017). Streaming refers to the act of grouping students into classes based on their intellectual and/ or academic endeavors in this study.

A large number of young generation's social and emotional development is occurring on internet and cell phones. Some studies proved that social media negatively affect the performance of students while according to some researches it improved the learning of its users and enhanced their communications skills. Keeping this in view the present research is being conducted to ascertain the impact of social media on youth.

\section{Materials and Methods}

The comparative study was conducted in urban and rural areas of Hisar district (Haryana). The sample comprised of four schools i.e. two higher secondary schools (one government and one private school) and two higher secondary schools from rural area (one government and one private school). Hisar district comprised of nine blocks, from which Hisar-I was randomly selected. From the selected schools, lists of $10+1$ and $10+2$ students were procured from school records. From these lists, 40 students (both male and female) were selected randomly from each school.

\section{Results and Discussion}

The results of the present investigation are described following on the basis of following subheadings:

Impact of social media on the health of students

Constraints faced by the students

\section{Impact of social media on the health of students}

The impact of social media on students has been presented under the following heads; (i) mental health, (ii) physical health (iii) social health.

Table 1 illustrated the perceived health affect of social media by the students. The perception on health was observed in terms of mental, physical and social health. Majority of the students 85.7 per cent agreed that social media affects their health. The Table further 
revealed that 83.7 per cent students agreed that it affects their mental health followed by physical health $(72.5 \%)$ and social health $(55.0 \%)$ respectively.

\section{Perceived impact of social media on mental health by students}

The data represented in Table 2 showed the impact of social media on the mental health as perceived by the students. To measure the impact of social media on health of the students to use three continuums scale i.e. severe, moderate and no effect was taken from both the areas.

\section{Perceived impact of social media on physical health by students (Direct)}

The data furnished in the Table 3 showed the perceived impact of social media on their physical health (direct) of the students. In rural area the maximum impact that affects severely was observed in headache $(80.0 \%)$ followed by ringxiety $(75.0 \%)$, eyestrain (70.0\%), decrease eyesight $(65.0 \%)$ and neck pain $(60.0 \%)$ respectively and least impact was observed in obesity $(20.0 \%)$.

\section{Perceived impact of social media on} physical health by students (Indirect)

The data presented in the Table 4 showed the perceived impact of social media on their physical health (indirect) by the students. In rural area the maximum impact that affects severely was observed in laziness $(60.0 \%)$ followed by weakness $(45.0 \%)$ respectively and least impact was found in irritating behaviour (25.0\%).

From urban area data showed the maximum impact that affects severely was observed in irritating behaviour $(80.0 \%)$ followed by laziness $(45.0 \%)$ and least impact was found in loss of weight $(20.0 \%)$.

\section{Perceived impact of social media on mental} health of students by parents and teachers

The data presented in Table 5 showed the perceived impact of social media on mental health of students by the parents/teachers on their wards.

The maximum impact observed by parents was on insomnia $(90.0 \%)$, stress and fear (85.0\% each), self-esteem (70.0\%) respectively and least impact was observed on depression (15.0\%). Maximum impact perceived by the teachers was on self-identity $(80.0 \%)$, self-esteem $(75.0 \%)$ and decrease self-confidence $(70.0 \%)$ respectively whereas minimum impact was observed on loss of appetite $(20.0 \%)$.

Impact of social media on physical health of students as perceived by parents and teachers

This data depicted in Table 6 showed the perceived impact of social media by parents and teachers on physical heath of the students on their wards.

Maximum impact observed by the parents was on laziness (90.0\%), headache and shoulder pain $(85.0 \%$ each), neck pain, eyes strain, sleep disturbance and irritating behavior $(75.0 \%$ each) and least impact as perceived by the parents was on obesity $(20.0 \%)$. As regards as impact perceived by the teachers, it was observed maximum on physical health of the students on laziness $(90.0 \%)$, sleep disturbance $(80.0 \%)$, irritating behavior $(75.0 \%)$, weakness $(70.0 \%)$ and headache $(20.0 \%)$ respectively.

Perceived impact of social media on social health of students by parents and teachers

The data presented in the Table 7 showed that the perceived impact of social media on social 
health of students by parents and teachers. Maximum impact perceived by the parents on social health of the student's on lack of interaction with parents $(75.0 \%)$, relatives $(70.0 \%)$ respectively and least impact was observed on lack of interaction with siblings $(30.0 \%)$.
Further maximum impact perceived by the parents on social health of the student's on lack of bonding $(70.0 \%)$ and least impact on aloofness (35.0\%). Whereas maximum impact perceived by the teachers on lack of bonding (55.0\%) and least impact on aloofness $(40.0 \%)$.

Table.1 Perceived health affect of social media by the students

\begin{tabular}{|c|c|c|c|c|c|}
\hline \multirow[t]{2}{*}{ Affect health } & \multicolumn{2}{|c|}{$\begin{array}{c}\text { Rural }(n=40) \\
f(\%)\end{array}$} & \multicolumn{2}{|c|}{$\begin{array}{c}\text { Urban }(n=40) \\
f(\%)\end{array}$} & \multirow[t]{2}{*}{$\begin{array}{c}\text { Total } \\
(\mathrm{n}=\mathbf{8 0}) \mathrm{f}(\%)\end{array}$} \\
\hline & Govt. & Private & Govt. & Private & \\
\hline Yes & $14(70.0)$ & $18(90.0)$ & $17(85.0)$ & $18(90.0)$ & $67(83.7)$ \\
\hline No & $06(30.0)$ & $2(10.0)$ & $3(15.0)$ & $2(10.0)$ & $13(16.2)$ \\
\hline \multicolumn{6}{|l|}{ If yes, type of affects } \\
\hline Mental & $14(70.0)$ & $18(90.0)$ & $17(85.0)$ & 18(90.0) & $67(83.7)$ \\
\hline Physical & $13(65.0)$ & $15(75.0)$ & $15(75.0)$ & $15(75.0)$ & $58(72.5)$ \\
\hline Social & $10(50.0)$ & $11(55.0)$ & $9(45.0)$ & $14(70.0)$ & $44(55.0)$ \\
\hline
\end{tabular}

Table.2 Perceived impact of social media on mental health by students

\begin{tabular}{|l|c|c|c|c|c|c|}
\hline \multicolumn{1}{|c|}{ Impacts } & \multicolumn{3}{c}{ Rural (n=20) f(\%) } & \multicolumn{3}{c|}{ Urban (n=20) f(\%) } \\
\cline { 3 - 6 } & Severe & Moderate & $\begin{array}{c}\text { No } \\
\text { effect }\end{array}$ & Severe & Moderate & $\begin{array}{c}\text { No } \\
\text { effect }\end{array}$ \\
\hline Insomnia & $12(60.0)$ & $5(25.0)$ & $3(15.0)$ & $16(80.0)$ & $4(20.0)$ & - \\
\hline Stress & $14(70.0)$ & $4(20.0)$ & $8(40.0)$ & $15(75.0)$ & $5(25.0)$ & - \\
\hline Loss of appetite & $15(75.0)$ & $4(20.0)$ & $1(5.0)$ & $12(60.0)$ & $5(25.0)$ & $2(10.0)$ \\
\hline Depression & $2(10.0)$ & $3(15.0)$ & $15(75.0)$ & $3(15.0)$ & $4(20.0)$ & $12(60.0)$ \\
\hline Loneliness & $16(80.0)$ & $4(20.0)$ & - & $16(80.0)$ & $6(30.0)$ & $2(10.0)$ \\
\hline Distraction & $4(20.0)$ & $7(35.0)$ & $9(45.0)$ & $12(60.0)$ & $2(10.0)$ & $6(30.0)$ \\
\hline Fear & $11(55.0)$ & $2(10.0)$ & $7(35.0)$ & $5(25.0)$ & $7(35.0)$ & $8(40.0)$ \\
\hline Anger & $9(45.0)$ & $7(35.0)$ & $4(20.0)$ & $13(65.0)$ & $4(20.0)$ & $3(15.0)$ \\
\hline $\begin{array}{l}\text { Decrease self } \\
\text { Confidence }\end{array}$ & $5(25.0)$ & $4(20.0)$ & $11(55.0)$ & $14(70.0)$ & $7(35.0)$ & $8(40.0)$ \\
\hline Decrease self esteem & $4(25.0)$ & $3(15.0)$ & $13(65.0)$ & $9(45.0)$ & $6(30.0)$ & $5(25.0)$ \\
\hline Self-identity & $8(40.0)$ & $9(45.0)$ & $3(15.0)$ & $10(50.0)$ & $6(30.0)$ & $4(20.0)$ \\
\hline $\begin{array}{l}\text { Change Attitude } \\
\text { about }\end{array}$ & & & & & & \\
\hline - Family & $6(30.0)$ & $9(45.0)$ & $5(25.0)$ & $7(35.0)$ & $8(40.0)$ & $5(25.0)$ \\
\hline - Self & $5(25.0)$ & $4(20.0)$ & $11(55.0)$ & $8(40.0)$ & $6(30.0)$ & $6(30.0)$ \\
\hline - Friends & $8(40.0)$ & $7(35.0)$ & $5(25.0)$ & $11(55.0)$ & $5(25.0)$ & $4(20.0)$ \\
\hline & & & & & & \\
\hline
\end{tabular}


Table.3 Perceived impact of social media on physical health by students (Direct)

\begin{tabular}{|l|c|c|c|c|c|c|}
\hline \multicolumn{1}{|c|}{ Impacts } & \multicolumn{3}{|c|}{ Rural (n=20) f(\%) } & \multicolumn{3}{c|}{ Urban (n=20) f(\%) } \\
\cline { 2 - 5 } & $\begin{array}{c}\text { Severe } \\
\mathbf{f}(\mathbf{\%})\end{array}$ & $\begin{array}{c}\text { Moderate } \\
\mathbf{f}(\%)\end{array}$ & $\begin{array}{c}\text { No effect } \\
\mathbf{f ( \% )}\end{array}$ & $\begin{array}{c}\text { Severe } \\
\mathbf{f}(\%)\end{array}$ & $\begin{array}{c}\text { Moderate } \\
\mathbf{f}(\%)\end{array}$ & $\begin{array}{c}\text { No effect } \\
\mathbf{f}(\%)\end{array}$ \\
\hline Neck pain & $12(60.0)$ & $5(25.0)$ & $03(15.0)$ & $6(30.0)$ & $12(60.0)$ & $2(10.0)$ \\
\hline Shoulder pain & $9(45.0)$ & $04(20.0)$ & $07(35.0)$ & $10(50.0)$ & $6(30.0)$ & $4(20.0)$ \\
\hline Wrist pain & $09(45.0)$ & $6(30.0)$ & $05(25.0)$ & $6(30.0)$ & $9(45.0)$ & $5(25.0)$ \\
\hline Eyes strain & $14(70.0)$ & $11(55.0)$ & $03(15.0)$ & $13(75.0)$ & $7(35.0)$ & - \\
\hline Headache & $16(80.0)$ & $3(15.0)$ & $01(5.0)$ & $6(30.0)$ & $13(65.0)$ & $1(5.0)$ \\
\hline Backache & $09(45.0)$ & $4(20.0)$ & $07(35.0)$ & $12(60.0)$ & $5(25.0)$ & $3(15.0)$ \\
\hline $\begin{array}{l}\text { Decrease } \\
\text { eyesight }\end{array}$ & $13(65.0)$ & $8(40.0)$ & $01(5.0)$ & $14(70.0)$ & $3(15.0)$ & $3(15.0)$ \\
\hline $\begin{array}{l}\text { Failure to eat } \\
\text { regular }\end{array}$ & $09(45.0)$ & $7(35.0)$ & $04(20.0)$ & $6(30.0)$ & $7(35.0)$ & $7(35.0)$ \\
\hline junk foods & $06(30.0)$ & $4(20.0)$ & $10(50.0)$ & $15(75.0)$ & $7(35.0)$ & $5(25.0)$ \\
\hline Overeating & $05(25.0)$ & $9(45.0)$ & $06(30.0)$ & $11(55.0)$ & $6(30.0)$ & $3(15.0)$ \\
\hline Obesity & $4(20.0)$ & $07(35.0)$ & $09(45.0)$ & $7(35.0)$ & $5(25.0)$ & $8(40.0)$ \\
\hline Lack of exercise & $06(30.0)$ & $8(40.0)$ & $06(30.0)$ & $6(30.0)$ & $4(20.0)$ & $10(50.0)$ \\
\hline Loss of appetite & $09(45.0)$ & $7(35.0)$ & $04(20.0)$ & $12(60.0)$ & $6(30.0)$ & $2(10.0)$ \\
\hline Loss of weight & $06(30.0)$ & $10(50.0)$ & $04(20.0)$ & $6(30.0)$ & $9(45.0)$ & $5(25.0)$ \\
\hline $\begin{array}{l}\text { Sleep } \\
\text { disturbances }\end{array}$ & $11(55.0)$ & $7(35.0)$ & $2(10.0)$ & $9(45.0)$ & $4(20.0)$ & $7(35.0)$ \\
\hline Selfitis & $09(45.0)$ & $7(35.0)$ & $4(20.0)$ & $14(70.0)$ & $11(55.0)$ & $5(25.0)$ \\
\hline Ringxiety & $15(75.0)$ & $5(25.0)$ & - & $12(60.0)$ & $2(10.0)$ & $3(15.0)$ \\
\hline $\begin{array}{l}\text { Neglect personal } \\
\text { Hygiene }\end{array}$ & $09(45.0)$ & $4(20.0)$ & $7(35.0)$ & $3(15.0)$ & $4(20.0)$ & $13(65.0)$ \\
\hline
\end{tabular}

Multiple responses

Table.4 Perceived impact of social media on physical health by students (Indirect)

\begin{tabular}{|l|c|c|c|c|c|c|}
\hline \multicolumn{1}{|c|}{ Variable } & \multicolumn{3}{|c|}{ Rural $(\mathbf{n = 2 0}) \mathbf{f}(\%)$} & \multicolumn{3}{c|}{ Urban (n=20) f (\%) } \\
\cline { 2 - 7 } & Severe & Moderate & $\begin{array}{c}\text { No } \\
\text { effect }\end{array}$ & Severe & Moderate & $\begin{array}{c}\text { No } \\
\text { effect }\end{array}$ \\
\hline Loss of weight & $7(35.0)$ & $4(20.0)$ & $9(45.0)$ & $4(20.0)$ & $9(45.0)$ & $7(35.0)$ \\
\hline Weakness & $9(45.0)$ & $6(30.0)$ & $5(25.0)$ & $6(30.0)$ & $4(20.0)$ & $10(50.0)$ \\
\hline Laziness & $12(60.0)$ & $4(20.0)$ & $4(20.0)$ & $9(45.0)$ & $8(40.0)$ & $3(15.0)$ \\
\hline Irritating behaviour & $5(25.0)$ & $14(70.0)$ & $1(5.0)$ & $16(80.0)$ & $4(20.0)$ & - \\
\hline
\end{tabular}

Multiple responses 
Table.5 Impact of social media on mental health of students as perceived by parents and teacher

\begin{tabular}{|c|c|c|c|c|}
\hline Impacts & $\begin{array}{c}\text { Parents }(n=20) \\
\text { f }(\%)\end{array}$ & Rank & $\begin{array}{c}\text { Teachers }(n=20) \\
f(\%)\end{array}$ & Rank \\
\hline \multicolumn{5}{|l|}{ Mental health } \\
\hline Insomnia & $18(90.0)$ & I & $13(65.0)$ & \\
\hline Stress & $17(85.0)$ & II & $09(45.0)$ & \\
\hline Loss of appetite & $12(60.0)$ & & $04(20.0)$ & \\
\hline Loneliness & $7(35.0)$ & & $11(55.0)$ & \\
\hline Fear & $17(85.0)$ & II & $09(45.0)$ & \\
\hline Anger & $13(65.0)$ & & $12(60.0)$ & \\
\hline Decrease self Confidence & $5(25.0)$ & & $14(70.0)$ & III \\
\hline Self-esteem & $14(70.0)$ & III & $15(75.0)$ & II \\
\hline Self-identity & $7(35.0)$ & & $16(80.0)$ & I \\
\hline Depression & $3(15.0)$ & & $05(25.0)$ & \\
\hline \multicolumn{5}{|l|}{ Change in Attitude } \\
\hline Family & $7(35.0)$ & & $09(45.0)$ & \\
\hline Self & $5(25.0)$ & & $07(35.0)$ & \\
\hline Friends & $11(55.0)$ & & $05(25.0)$ & \\
\hline
\end{tabular}

Table.6 Perceived impact of social media on physical health of students by parents and teachers

\begin{tabular}{|c|c|c|c|c|}
\hline Impacts & Parents $(n=20) f(\%)$ & Rank & Teachers $(n=20)$ f $(\%)$ & Rank \\
\hline \multicolumn{5}{|l|}{ Physical health } \\
\hline Neck pain & $15(75.0)$ & III & $10(50.0)$ & \\
\hline Shoulder pain & $17(85.0)$ & II & $11(55.0)$ & \\
\hline Wrist pain & $14(70.0)$ & & $9(45.0)$ & \\
\hline Eyes strain & $15(75.0)$ & III & $13(65.0)$ & \\
\hline Headache & $17(85.0)$ & II & $4(20.0)$ & \\
\hline Backache & $12(60.0)$ & & $6(30.0)$ & \\
\hline Decrease eyesight & $7(35.0)$ & & $12(60.0)$ & \\
\hline Failure to eat regular & $9(45.0)$ & & $11(55.0)$ & \\
\hline Obesity & $4(20.0)$ & & $7(35.0)$ & \\
\hline Lack of exercise & $6(30.0)$ & & $6(30.0)$ & \\
\hline Loss of appetite & $8(40.0)$ & & $5(25.0)$ & \\
\hline Loss of weight & $14(70.0)$ & IV & $9(45.0)$ & \\
\hline Sleep disturbances & $15(75.0)$ & III & $16(80.0)$ & II \\
\hline Selfitis & $12(60.0)$ & & $12(60.0)$ & \\
\hline Ringxiety & $14(70.0)$ & IV & $9(45.0)$ & \\
\hline Neglect personal hygiene & $9(45.0)$ & & $11(55.0)$ & \\
\hline Weakness & $7(35.0)$ & & $14(70.0)$ & IV \\
\hline Laziness & $18(90.0)$ & I & $18(90.0)$ & I \\
\hline Irritating behaviour & $15(75.0)$ & & $15(75.0)$ & III \\
\hline
\end{tabular}

Multiple responses 
Table.7 Perceived impact of social media on social health of students by parents and teachers

\begin{tabular}{|l|c|c|c|c|}
\hline \multicolumn{1}{|c|}{ Impacts } & $\begin{array}{l}\text { Parents }(\mathbf{n = 2 0}) \\
\mathbf{f}(\boldsymbol{\%})\end{array}$ & Rank & $\begin{array}{c}\text { Teachers } \\
(\mathbf{n = 2 0 )} \mathbf{f}(\boldsymbol{\%})\end{array}$ & Rank \\
\hline Lack of interaction with & & & & \\
\hline Parents & $15(75.0)$ & I & $9(45.0)$ & \\
\hline Friends & $9(45.0)$ & & $5(25.0)$ & \\
\hline Siblings & $6(30.0)$ & & $12(60.0)$ & II \\
\hline Neighbour & $12(60.0)$ & III & $11(55.0)$ & III \\
\hline Relatives & $14(70.0)$ & II & $15(75.0)$ & I \\
\hline Teachers & $9(45.0)$ & & $7(35.0)$ & \\
\hline Lack of participation in social event & & & & \\
\hline Aloofness & $7(35.0)$ & & $8(40.0)$ & \\
\hline lack of bonding & $14(70.0)$ & II & $11(55.0)$ & \\
\hline
\end{tabular}

Multiple responses

Table.8 Attitude of rural parents/teachers towards social media

\begin{tabular}{|c|c|c|c|c|}
\hline & \multicolumn{2}{|c|}{ Rural } & \multicolumn{2}{c|}{ Urban } \\
\hline Attitude & $\begin{array}{c}\text { Parents (n=80) } \\
\mathbf{f}(\mathbf{\%})\end{array}$ & $\begin{array}{c}\text { Teachers }(\mathbf{n = 1 1}) \\
\mathbf{f}(\mathbf{\%})\end{array}$ & $\begin{array}{c}\text { Parents } \\
(\mathbf{n = 8 0} \mathbf{f}(\mathbf{\%})\end{array}$ & $\begin{array}{c}\text { Teachers }(\mathbf{n = 1 4}) \\
\mathbf{f}(\mathbf{\%})\end{array}$ \\
\hline Favorable (72-56) & $13(16.2)$ & $7(63.6)$ & $52(65.0)$ & $11(78.5)$ \\
\hline Neutral (55-39) & $3(3.7)$ & $1(9.0)$ & $8(10.0)$ & $1(7.1)$ \\
\hline Unfavorable (38-22) & $64(80.0)$ & $3(27.4)$ & $20(25.0)$ & $2(14.3)$ \\
\hline Total & $80(100.0)$ & $11(100.0)$ & $80(100.0)$ & $14(100.0)$ \\
\hline
\end{tabular}

Table.9 Constraints/fears reported by respondents while using social media

\begin{tabular}{|l|c|c|c|}
\hline Constraints & $\begin{array}{c}\text { Rural (n=80) } \\
\mathbf{f ( \% )}\end{array}$ & $\begin{array}{c}\text { Urban (n=80) } \\
\mathbf{f ( \% )}\end{array}$ & $\begin{array}{c}\text { Total (n=160) } \\
\mathbf{f}(\mathbf{\%})\end{array}$ \\
\hline Face any constraint & \multicolumn{3}{|c|}{} \\
\hline Yes & $80(100)$ & $80(100)$ & $160(100)$ \\
\hline No & - & - & - \\
\hline Personal & & & \\
\hline Don't know how to use & $21(26.3)$ & $13(16.3)$ & $34(20.5)$ \\
\hline Privacy & $15(18.7)$ & $9(11.3)$ & $24(14.4)$ \\
\hline Divert attention & $23(28.7)$ & $29(36.3)$ & $52(31.3)$ \\
\hline Parents do not allow & $65(81.3)$ & $62(77.5)$ & $127(79.3)$ \\
\hline Lack of time & $24(30.0)$ & $29(36.2)$ & $53(33.1)$ \\
\hline Fear of online sexual predators & $5(6.25)$ & $07(8.7)$ & $12(7.2)$ \\
\hline Originality and copyright issue & $11(13.7)$ & $15(18.7)$ & $26(15.6)$ \\
\hline Unproductive behavior & $13(31.3)$ & $43(53.7)$ & $68(40.8)$ \\
\hline Neglect family responsibility & $18(22.5)$ & $31(38.7)$ & $49(29.4)$ \\
\hline Exposure to unwanted material & $8(10.0)$ & $19(23.7)$ & $33(20.6)$ \\
\hline Social & & & \\
\hline & & & \\
\hline
\end{tabular}




\begin{tabular}{|l|c|c|c|}
\hline Bullying & $15(18.7)$ & $11(13.7)$ & $26(15.6)$ \\
\hline Virtual relation & $29(36.3)$ & $33(41.3)$ & $52(31.2)$ \\
\hline Antisocial behavior & $10(12.5)$ & $05(6.3)$ & $15(9.0)$ \\
\hline Online abuse & $18(22.5)$ & $22(27.5)$ & $40(25.0)$ \\
\hline Unwanted interaction & $14(17.5)$ & $32(40.0)$ & $46(28.7)$ \\
\hline Cyber crime & $09(11.3)$ & $12(15.0)$ & $21(12.6)$ \\
\hline Negative comment & $21(26.3)$ & $29(36.3)$ & $51(30.6)$ \\
\hline Technical & $37(46.3)$ & $15(18.7)$ & $52(31.2)$ \\
\hline Electricity failure & $24(30.0)$ & $29(36.2)$ & $53(33.1)$ \\
\hline Hacking of data & & \\
\hline Economic & $39(48.7)$ & $22(27.5)$ & $61(36.6)$ \\
\hline Non availability of gadget's & $55(68.7)$ & $35(43.7)$ & $90(56.2)$ \\
\hline Non availability of net facility & $51(63.7)$ & $32(40.0)$ & $83(49.8)$ \\
\hline Costly & & & \\
\hline
\end{tabular}

Multiple responses

\section{Attitude of rural and urban parents/teachers towards social media}

The data in Table 8 showed the attitude of rural parents and teacher's towards social media responses were obtained on three point continuum i.e. favorable, neutral and not favorable. Eighty per cent of parents had notfavorable attitude towards social media followed by 16.2 per cent parents had favorable attitude while 3.7 per cent of parents had neutral attitude towards social media.

Whereas 63.6 per cent of teachers had favorable attitude towards social media followed by 27.4 per cent of teachers had not favorable attitude while only nine per cent of teachers had neutral attitude towards social media.

The data in the Table 27 showed the attitude of urban parents and teachers towards social media. Sixty five per cent of parents had favorable attitude towards social media followed by twenty five per cent parents had unfavorable attitude while only ten per cent parents had neutral attitude towards social media.

The data in the Table 8 also showed the attitude of urban parents and teachers towards social media. Sixty five per cent of parents had favorable attitude towards social media followed by twenty five per cent parents had not-favorable attitude while only ten per cent parents had neutral attitude towards social media. On the other hand, 78.5 per cent of teachers had favorable attitude towards social media followed by non-favorable attitude $(14.3 \%)$ and neutral attitude $(7.1 \%)$ respectively.

\section{Constraints faced by the students}

This section comprises information about the constraints faced by the students while using social media (Table 9). Constraints were studied on four aspects i.e. personal, social, technical and economic.

Under personal aspect maximum constraints was observed by the students was that parents do not allow (79.3\%) and least constraint was observed fear of online sexual predators (7.5\%), under social aspect constraints was maximum constraints was observed in negative comments $(30.0 \%)$ and least constraints was observed in antisocial behavior $(9.0 \%)$, under technical aspect constraints maximum constraints was observed in hacking of data $(33.1 \%)$ and least constraints was observed electricity failure 
(31.2\%) and the last economic aspect maximum constraints was observed in the non-availability of net facility $(56.2 \%)$ and least was observed in non-availability of gadgets $(36.6 \%)$.

In conclusions to solve the constraints faced by the students, parents should themselves equipped with computer skill so that they can guide their children properly in terms of how, when and what type of social media they should use in order to show the right path to their children. Hence it is concluded that children should be well educated about social media either from parents, teachers or trained social media experts. Parents should have friendly relations with their child so that all type of things they can share between themselves. Students should participate in outdoor activities to have good physical health.

\section{References}

Asur, S. and Huberman, B.A. (2010). Predicting the future with social media. Social Computing Lab: HP Labs, Palo Alto, California. 7(1): 1-8.

Boyd, D. (2010). Taken out of context: American teen sociality in networked public berkeley, CA: University of California.

Camilia, O., Sajoh, I., and Dalhatu, B. (2013). The Effect of social networking sites usage on the studies of Nigerian students. The international journal of engineering and science. 2(7): 39- 46.

Kaur, R. and Bashir, H. (2015). Impact of social media on mental health of adolescents. International Journal of Education. 5(1): 2229.
Kimberly, M. (2017). Remaining safe and avoiding dangers online: a social media $\mathrm{Q}$ \& A with kimberly mitchell. The Prevention Researcher. 17(1): 7-10.

Kumar N. and Sharma S. (2017). Survey analysis on the usage and impact of whatsapp messenger Global. Journal of Enterprise Information System. 8(3): 94101.

Lou, L.L., Yan, Z., Nickerson, A. and McMorris, R. (2012). An examination of the reciprocal relationship of loneliness and Facebook use among first-year college students. Journal of educational computing research. 46(1): 105-117.

Madden, M. and Zickhur, K. (2011). 65\% of online adults use social networking sites: Women maintain their foothold on S.S use and older Americans are still coming aboard. Pew internet and American life Project. http://pewinternet.org/Reports/2011/Social Networking-Sites.aspx.

Malathy, M. and Rani, M. (2018). How social media creates an impact in our mental, physical health and culture - portrayal of social mobile media culture technology in India. Portrayal of Social Issues in Literature and Media. 18(3): 32-36

Prabandari, K. and Yuliati, L. N. (2016). The Influence of Social Media Use and Parenting Style on Teenagers' Academic Motivation and Academic Achievement. Journal of Child Development Studies. $1(1): 40-49$.

Shahzad. K. (2015). Impact of social networking sites on students. Abasyn Journal of Social Science,5(2), 52-71.

Thurstone, L.L. (1946). "Comment" in American Journal of Sociology, 52, pp.39-50.

\section{How to cite this article:}

Sanyogita, Poonam Kundu and Aman. 2020. Perceived Impact of Social Media on Higher Secondary School Students. Int.J.Curr.Microbiol.App.Sci. 9(10): 4006-4014. doi: https://doi.org/10.20546/ijcmas.2020.910.460 